\title{
marges Marges
}

revue d'art contemporain Revue d'art contemporain

05 | 2007

L'exposition sous toutes ses formes

\section{Entretien entre François Piron et Rozen Canévet}

Interview of François Piron by Rozenn Canévet

\section{Rozenn Canévet et François Piron}

\section{(2) OpenEdition}

Journals

Édition électronique

URL : http://journals.openedition.org/marges/712

DOI : $10.4000 /$ marges.712

ISSN : 2416-8742

\section{Éditeur}

Presses universitaires de Vincennes

\section{Édition imprimée}

Date de publication : 15 juin 2007

Pagination : 99-111

ISBN : 978-2-84292-249-8

ISSN : 1767-7114

Référence électronique

Rozenn Canévet et François Piron, « Entretien entre François Piron et Rozen Canévet », Marges [En ligne], 05 | 2007, mis en ligne le 15 juin 2008, consulté le 20 avril 2019. URL : http:// journals.openedition.org/marges/712 ; DOI : 10.4000/marges.712

Ce document a été généré automatiquement le 20 avril 2019

(C) Presses universitaires de Vincennes 


\title{
Entretien entre François Piron et Rozen Canévet
}

\author{
Interview of François Piron by Rozenn Canévet
}

Rozenn Canévet et François Piron

Des œuvres de Carsten Höller, Laurent Montarron, Ann Veronica Janssens, Eija-Liisa Ahtila, Jim Shaw, François Curlet, Rodney Graham, Claude Lévêque, Simone Decker, Laurent Grasso, Dominique Petitgand, Peter Fischli et David Weiss, Tacita Dean, Michel Blazy, Christophe Berdaguer \& Marie Péjus occupaient l'espace muséal, créant des correspondances aléatoires les unes avec les autres, impliquant physiquement le visiteur. Jouant sur l'incertitude, évitant tout critère dogmatique, «Subréel » a généré un mode d'exposition sur la mise à l'épreuve dont la forme altère plus qu'elle ne dénoue. À travers le filtre de cette expérience, François Piron explique sa conception de l'exposition en tant que curateur.

Rozenn Canévet: Comment définiriez-vous la thématique de l'exposition «Subréel » que vous avez organisée au m.a.c. de Marseille en 2002 ?

François Piron: «Subréel » est une exposition qui n'a pas été pensée selon une perspective thématique. Je n'ai rien contre les expositions thématiques - elles ne sont pas forcément synonymes d'expositions illustratives - et «Subréel » aurait tout à fait pu être élaborée autrement, mais elle a d'abord été pensée comme un parcours entre des œuvres choisies assez subjectivement, par Nathalie Ergino et moi-même, en pingpong. Elle s'est constituée par les rapports instaurés entre un noyau dur d'œuvres et d'autres qui se sont ajoutées en complément ou en réaction, venant muscler, brouiller ou étoiler les significations qui s'échafaudent par l'expérience de ces œuvres. Chaque œuvre joue un "rôle" dans une dramaturgie générale. Une exposition se construit souvent sur des questions extrêmement générales qui, à partir du moment où on les exemplifie à travers une œuvre précise, redeviennent spécifiques. Dans ce cas, des questions sur le rapport du spectateur à l'œuvre, sur ce que serait la beauté aujourd'hui, ou des questions plus précises sur la relation entre ennui et fascination, ou 
encore sur la définition d'un imaginaire envisagé sur un mode physiologique... sont présentes, mais ne constituent jamais des résolutions de l'exposition «Subréel ». Si on l'aborde sous l'angle de l'illustration ou du développement d'un thème, la présence de certaines œuvres semble très incongrue. L'enjeu était au contraire d'empêcher toute appréhension et compréhension rapides de l'exposition.

R.c. : Dans le choix des œuvres, au-delà de leurs spécificités, on perçoit un intérêt général pour les jeux de perception. Par ailleurs, dans le texte du catalogue, dans la partie intitulée «Prolégomènes », vous vous référez à la notion d'imaginaire définie par Clément Rosset. À savoir: "Un réel légèrement décalé par rapport à son espace et son temps propres. ». Imaginaire, précise-t-il, «qui n'existe qu'à la faveur de l'imagination et ne saurait donc jamais être le résultat d'une perception directe du réel. ». Vous vous interrogez alors sur le mode de perception directe du réel en utilisant les termes «d'encodages complexes du mental » et « d'extra-corporalité ». Avez-vous réuni ces œuvres en fonction de ce coefficient de décalage avec le réel ?

F.p.: La notion d'altération, qui revient souvent dans le texte du catalogue, est un fil conducteur important. Comment passe-t-on de quelque chose de familier à une "altérité »? Nous étions intéressés à éprouver et faire éprouver une forme " d'inquiétante étrangeté », avec des œuvres qui questionnent l'imaginaire et ses conditionnements, ses contraintes. Le rêve, la démence, mais aussi l'hypnose, l'ivresse, la stimulation rétinienne. Ces œuvres - même si elles jouent parfois une forme d'efficacité directe de stimulus du spectateur, dans le prolongement de l'art optique et cinétique par exemple - adoptent néanmoins une attitude souvent distanciée, jouant de l'humour ou au contraire de la froideur scientifique, de l'accumulation systémique. Nous étions également intéressés par des œuvres qui se révèlent autres que leur apparence, qui contiennent une forme de camouflage formel, en empruntant des formes reconnaissables de l'art moderne et contemporain. 


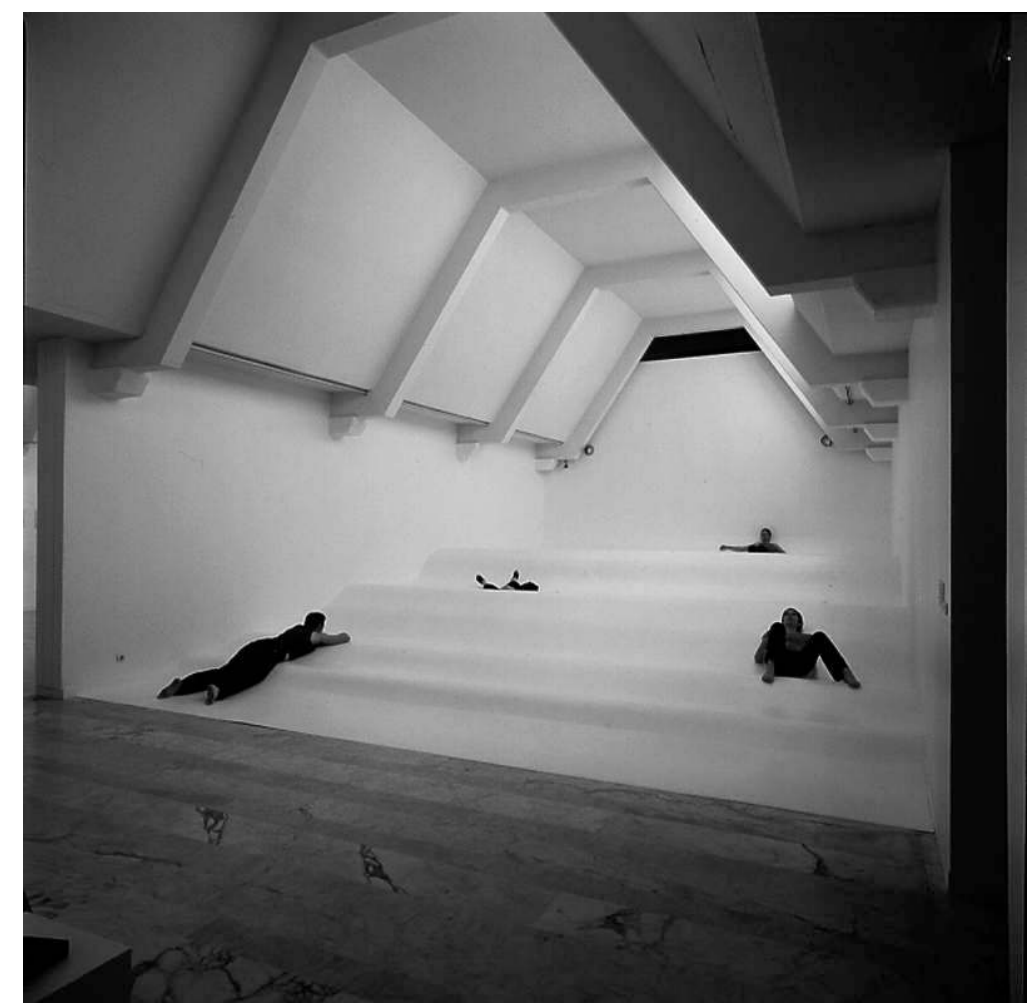

(Le Pavillon de chasse), adhésif double face, métal, dimensions variables, 1998-2002.

R.c. : Etes-vous en accord avec l'idée répandue d'une réappropriation (plus ou moins transparente) des expérimentations scientifiques sur les modes perceptifs dans l'art d'aujourd'hui?

F.p. : Prenons l'exemple de Carsten Höller, dont la critique d'art mythologise beaucoup le passé de biologiste et d'entomologiste. La pièce présentée dans l'exposition «Subréel» - Light Corner - repose sur un principe extrêmement simple, et scientifiquement anachronique. C'est l'agrandissement d'un dispositif de test de l'épilepsie qui date des années 1930 , basé sur un clignotement de lumière à la fréquence de $7,8 \mathrm{~Hz}$ pour détecter des réactions épileptiques. L'efficacité de ce dispositif agrandi ne doit pas faire oublier qu'il s'agit avant tout d'un choix formel et d'un commentaire sur l'art optique. C'est aussi selon moi un commentaire sur une relation refoulée entre un certain positivisme scientiste et un système de croyance, un mysticisme qui a toujours été intrinsèquement lié à l'acquisition de nouvelles technologies et au développement de la connaissance. On connaît ces photographes qui, au $19^{\mathrm{e}}$ siècle, cherchaient à photographier la pensée, l'aura, l'âme. Thomas Edison était un temps persuadé qu'on pourrait communiquer avec les morts grâce au téléphone. Ce fond de croyance a persisté au sein de la modernité rationnelle et on aurait tort de penser qu'il s'est évaporé aujourd'hui. Je crois que des artistes comme Carsten Höller, Rodney Graham, Laurent Montaron ou François Curlet font émerger à nouveau cette relation étrange qu'il y a entre la croyance et l'impératif rationaliste ce qui, du coup, devient aussi un commentaire sur l'art moderne. À la sentence minimaliste de Franck Stella, "ce que vous voyez est ce que vous voyez ", ils réintroduisent un certain système de croyance qui instaure un "nuage de fiction ", comme dit Philippe Parreno, autour des formes visibles. C'est une relecture des fondations de l'art contemporain, si l'on 
considère par exemple la première des «sentences" de Sol Lewitt: «les artistes conceptuels sont plus mystiques que rationalistes. ».

R.c. : Les artistes de «Subréel » appartiennent à une génération qui succède, si l'on veut, à une génération qui a fait du concept de divertissement une problématique dominante. II n'y a pas si longtemps, le cynisme était roi et la critique acerbe. Aujourd'hui, face à l'ennui contemporain, ces artistes proposent-ils d'autres alternatives qu'une prise de position politique affirmée comme, par exemple, une réinvention de l'imaginaire par de nouvelles formes d'échanges entre le spectateur et l'œuvre?

F.p.: Tout le monde connaît maintenant les bases de la psychanalyse et de la psychiatrie, il est impossible d'être dans l'onirisme par rapport au fonctionnement de l'imaginaire. On sait qu'il n'est pas une plus-value de l'âme, ni une échappatoire au réel. Il est beaucoup plus intéressant de l'envisager comme Clément Rosset, c'est-à-dire comme une part du réel et comme quelque chose qui s'ouvre partout dans la réalité.

Cette génération récente d'artistes est consciente que l'art et le musée font partie de la sphère du divertissement et n'a plus la prétention de se positionner en-dehors. Ils travaillent plutôt à instiller un pourcentage de résistance. J'emploie ce terme dans sa signification d'ordre physique, une sorte de solidité pneumatique étanche à l'appréhension immédiate. Une des formes de cette résistance s'instaure par l'ambiguïté. Ce que Carsten Höller appelle le doute, c'est le « double tranchant » et c'est ce que je voulais faire éprouver dans "Subréel», avec des œuvres qui chacune élaborent les propres termes de leur ambiguïté. Par exemple, l'œuvre de François Curlet, Rorschach Saloon, ironise sur la convivialité en instaurant un dispositif d'autorité qui empêche toute appropriation immédiate. Elle joue sur une immédiateté de reconnaissance des formes (la porte de saloon, le dispositif des bancs qui renvoie au divan du psychanalyste), et sur l'incongruité de sa présence dans le musée, sur l'expectative intrigante d'une utilisation possible, mais simplement suggérée (la disponibilité des bouteilles de whisky et de vodka, insérées dans les bancs). C'est une pièce qui renvoie également au dispositif muséal (des bancs en tant que sculpture), tout en le mettant en crise, puisque, grâce à elle, on peut potentiellement parcourir l'exposition en état d'ivresse.

Le film Le Rayon vert de Tacita Dean pose une autre ambiguïté. Il s'énonce comme la captation objective d'un phénomène météorologique, dans une approche documentaire, et pourtant, au final, le résultat est une carte postale aussi fascinante qu'ennuyeuse, un plan fixe de coucher de soleil qui paraît interminable. Cette pièce suscite l'attente et l'expectative jusqu'à un état hypnotique qui fait que le spectateur rate quasiment à chaque fois l'instant du rayon vert qui est censé être l'événement du film. Résultat : le spectateur ne sait plus exactement ce à quoi il est confronté et il est amené à se poser la question de ce que lui-même recherche dans sa relation à l'œuvre d'art. 


\section{Claude Lévêque, Sans titre, vidéo}

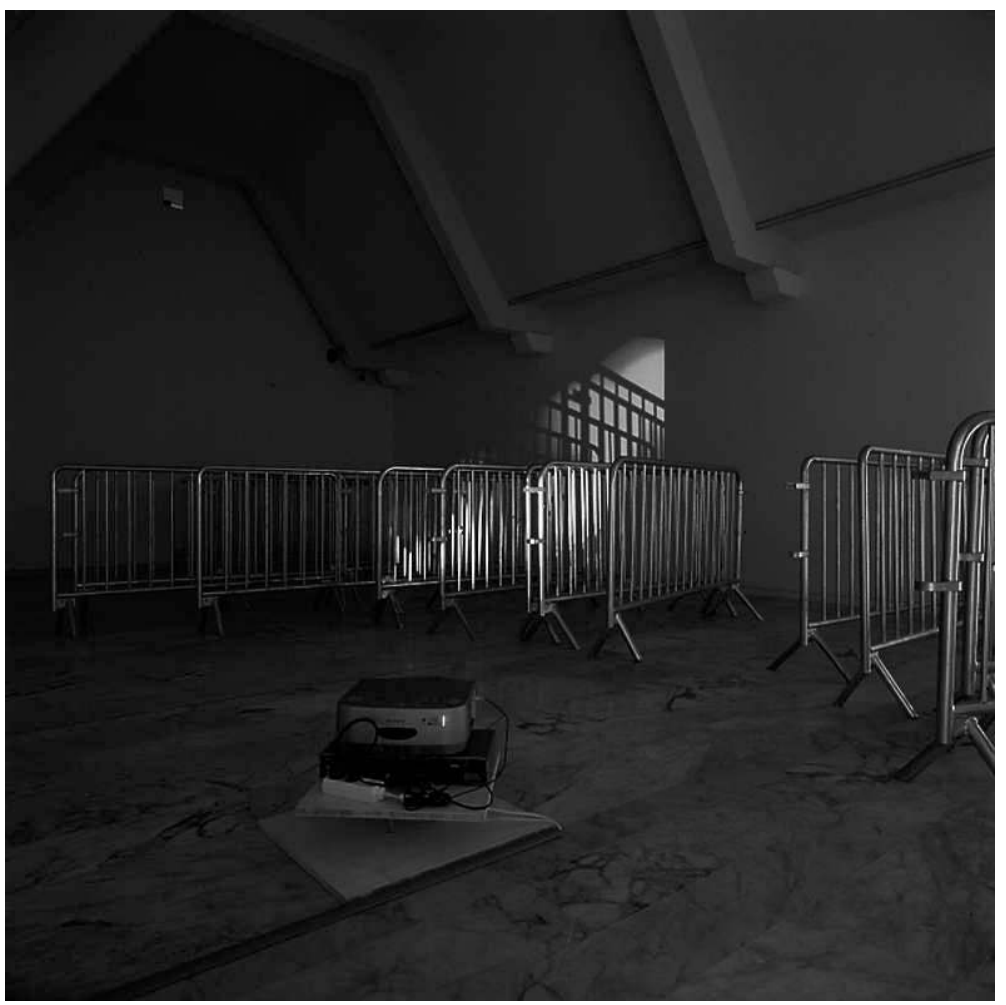

Plateau tournant, barrières de sécurité chromées, dimension variables, 1997.

\section{Ann Veronica Jansens, Représentation d'un corps rond}

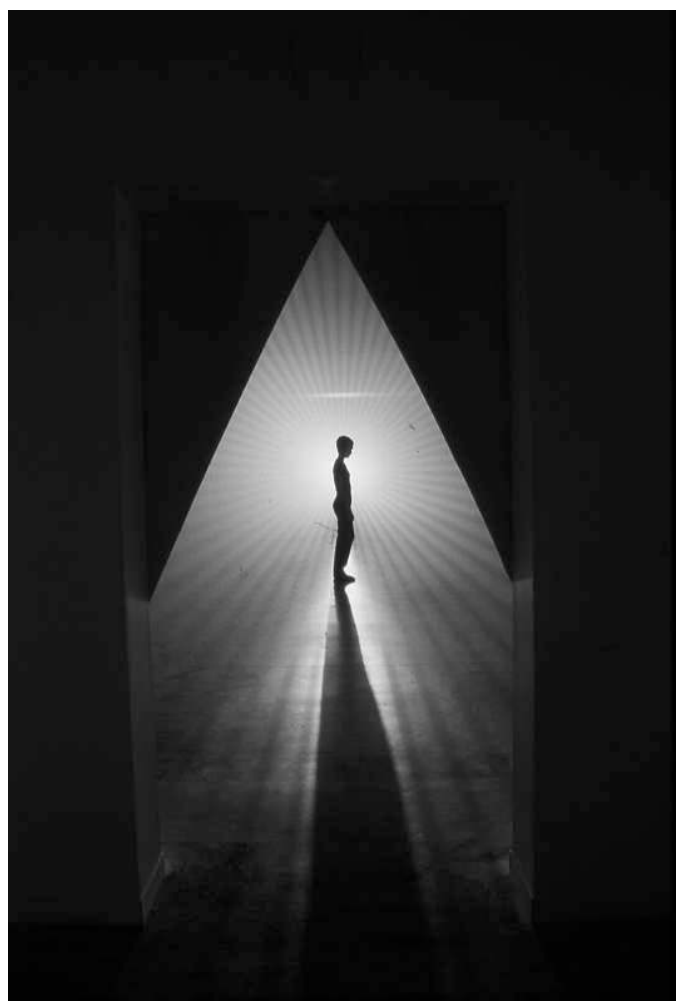

Projecteur cyberlight, brouillard articiel, dimensions variables, 1996-2001. 
R.c. : Dans le texte du catalogue, vous parlez de «coefficient d'irréalité ». Est-ce que cela rejoint l'idée d'une forme de conscience de soi qui serait complètement engourdie avec, par exemple, la pièce de Tacita Dean et qui, par ailleurs, serait réactivée chez le spectateur, physiquement et mentalement, par un jeu de contraste sur les modes de réception de l'œuvre? Toutes ces pièces provoquent des turbulences perceptives pour le spectateur dont le corps devient stimulé, exposé et activé. N'en résulte-t-il pas une conscience de soi augmentée - parce que mise à l'épreuve - chez le récepteur de l'œuvre ?

F.p.: Cette idée de la conscience de soi du spectateur est quelque chose qui doit être travaillé par le curateur au sein de la dramaturgie de l'exposition. Il y avait délibérément une indéfinition de l'exposition avec le choix de ce titre peu explicite, "Subréel ». Ainsi, dès l'entrée de l'exposition, le spectateur était jeté dans quelque chose d'indéfini et ce, sans autre forme de procès. J'ai tenu à ce qu'il n'y ait pas de texte explicatif, que chaque œuvre soit complètement autonome et que, même pour les pièces où une certaine interactivité était possible, il n'y ait rien d'indiqué afin que tout soit laissé au libre-arbitre du spectateur. Pour les pièces à fonctionnement potentiellement "participatif», le moment qui m'intéresse est celui du choix du spectateur, son hésitation entre plusieurs comportements.

L'effet d'autorité intrinsèque du musée (pour moi intéressante) où l'œuvre est toujours fétichisée, où la relation est individuelle, était aussi un paramètre pour cela. Un certain nombre d'œuvres incitaient le spectateur à assumer des choix : attendre ou pas, agir ou pas, regarder ou pas...

R.c. : Cela rejoint l'idée de la transgression des codes comportementaux qui jouerait sur une hypothétique mise en péril de l'autorité institutionnelle mais aussi de l'œuvre ou de soi?

F.p. : Si vous voulez, mais avec encore une fois beaucoup de distance. Par exemple, la question du danger (l'ébriété possible chez Curlet, la stimulation épileptique chez Höller, les drogues disponibles chez Berdaguer \& Péjus) est présentée délibérément comme factice. C'est évidemment un jeu dont chaque spectateur accepte de partager les règles ou non. Pour Carsten Höller, nous avons été obligés de prévenir les personnes sujettes à l'épilepsie de ne pas s'exposer à cette œuvre. Cela reste spéculatif car selon moi, ce n'est pas l'idée d'un réel danger qui est intéressante mais plutôt la sensation de basculement, d'altération. Le temps de la critique institutionnelle frontale est révolu.

R.c. : N'est-ce pas aussi un test sur les limites acceptées du comportement du visiteur et, conséquemment, une manière de le provoquer, de perturber ses habitudes pour favoriser une émergence, un « éveil des sens » physique et intellectuel ?

F.p.: Toute œuvre d'art est un "éveil des sens ", comme vous dites. L'activité, avant tout mentale, du spectateur m'intéresse : le confronter à cinquante dessins de rêve de Jim Shaw, par exemple, est une manière de stimuler un dilemme. Le luxe de détails contenus dans un seul de ces dessins provoque un effort d'attention. Il est presque impossible à soutenir si on multiplie cet effort par cinquante et cela met en crise le regard, la question de l'attention - de manière physique également. Pour moi, la relation à l'art n'est pas une expérience sociale mais une expérience individuelle. L'exposition doit rester un espace de doute, d'irrésolution, un moment d'incertitude. Je déteste les dispositifs didactiques qui expliquent les œuvres au spectateur, même si certaines informations doivent être distillées parfois. Il est important de savoir que Kanalvideo de Fischli et Weiss est un film ready-made enregistré par une caméra téléguidée dans une canalisation d'égout. Mais finalement ce n'est qu'un niveau de compréhension du film dont on pourrait dans d'autres contextes éventuellement se 
passer. Je m'intéresse davantage aux histoires que génèrent les œuvres d'art qu'à celles qui relèvent de leur généalogie.

\section{R.c. : Une histoire dans le sens d'un mythe ou d'une anecdote ?}

F.p. : Ce n'est pas anecdotique. C'est l'idée que l'art produit des histoires. Cela renvoie aussi au mythe et aux systèmes de croyance. Gianni Motti a réalisé à Zurich une rétrospective de son travail pour laquelle il a construit un labyrinthe avec des gardiens - postés à différents endroits de ce parcours - qui racontent ses œuvres aux visiteurs. C'est aussi le principe de la rétrospective récente de Rirkrit Tiravanija en Europe. C'est une intéressante production de mythologie, par transmission orale. Philippe Parreno, par exemple, qui a écrit un texte pour cette rétrospective de Tiravanija, est un créateur de mythologies. On ne peut que spéculer sur les œuvres de Parreno, car elles sont si ténues, si peu autonomes, qu'elles ont à peine un statut. Lors de son exposition au Musée d'art moderne de la ville de Paris, il a complètement dés-autonomisé les œuvres et a fait de l'exposition une œuvre. Le moment où le visiteur marche entre deux pièces devient le moment de l'œuvre, comme le silence de Mozart !

R.c. : Cela rejoint ce que vous disiez à propos de la circulation entre les œuvres : la prise en compte du parcours est aussi primordiale que la confrontation autonome spectateur / œuvre. Comme si, finalement, vous jouiez d'une sorte de trafic d'influences de l'ensemble des œuvres de l'exposition sur la réception d'une œuvre par le spectateur.

F.p. : Exactement. On ne s'occupe pas assez du corps du spectateur, il est complètement nié dans la plupart des expositions.

R.c. : Parfois, la prise en compte du corps passe par un mode presque agressif, par une stimulation excessive.

F.p.: Agresser le spectateur n'est pas un objectif mais cela peut être un mode opératoire. On peut considérer que la pièce de Carsten Höller est une pièce agressive. En fait, elle était surtout violente vis-à-vis des autres œuvres plus qu'envers les spectateurs. Elle rayonnait partout dans le musée qui vibrait de ses pulsations lumineuses. Et certains artistes de l'exposition étaient ennuyés car cela nuisait à leur propre autonomie.

Une exposition repose sur la mise en relation des œuvres. La contamination est une approche possible. Un des projets de Laurent Montaron - qui n'a finalement pas été réalisé - était de diffuser une odeur d'éther qui aurait flotté dans tout le musée. Christophe Berdaguer et Marie Péjus travaillent aussi la diffusion de substances invisibles, impalpables, pour certaines de leurs pièces, par exemple des phéromones. Ils se positionnent vraiment comme des artistes et non comme des designers ou des architectes, et en ce sens, encore une fois, l'aspect spéculatif et la croyance sont extrêmement importants. Autrement dit, la scientificité de la chose - et je rejoins encore une fois cette idée de coefficient d'irréalité - n'est pas un but en soi. Lorsque Berdaguer et Péjus réalisent un circuit d'alimentation en eau additionnée de somnifères, le but n'est pas l'efficacité du produit. Il s'agit d'ailleurs d'un effet placebo car la dose est trop infinitésimale pour que celui qui la consomme en ressente les effets. L'objectif n'est pas de chloroformer le spectateur mais de produire cette croyance. Pour ce faire, il est nécessaire d'instaurer un équilibre. Si c'est seulement de l'eau et que le cartel seul produit la fiction, ce n'est sans doute pas suffisant. Il faut qu'il y ait un degré de crédibilité scientifique même si le but n'est pas de le rendre efficace. C'est là la différence avec les réalisations de Philippe Rahm, qui, lui, semble curieusement convaincu des effets physiologiques de ses expériences. 
R.c. : Dans son article intitulé «Expanded Arts, Expanded Visions [...]», Jean-Christophe Royoux revient sur le concept d'élargissement apparu dans les années soixante. II parle d'un projet dialogique entre l'œuvre et le spectateur dont le corps deviendrait exposé. En regard de certaines œuvres de cette décennie, beaucoup semblent réactualiser ce concept jusqu'à en faire le paradigme d'un concept artistique (l'esthétique physiologique de Rahm ou celle du doute de Höller) dont le «champ de bataille » implique le corps du spectateur. Qu'en pensez-vous?

F.p. : Je ne pense pas que l'on puisse circonscrire ce genre de théories à une esthétique ou à une époque particulière. On peut tout à fait considérer le Grand Verre de Duchamp de ce point de vue. Les champs de bataille m'intéressent moins que les approches transhistoriques permettant des relectures subjectives, délibérément partiales et incomplètes, mais affirmatives d'une position. Ce qui m'intéresse, c'est une application spéculative de cette théorie sur des œuvres qui, a priori, n'ont strictement rien à voir avec cela. On peut dire que le Grand Verre de Duchamp est l'exemple le plus probant de l'autonomie de l'œuvre d'art, au sens d'une œuvre totalement fermée, hermétique, et à la fois totalement ouverte, spéculative. Par exemple, cela m'intéressait d'éprouver les thèses de Jean-Christophe Royoux avec l'œuvre de Gerhard Richter, plutôt qu'avec Pierre Huyghe, justement parce que Richter ne travaille pas là-dessus. D'un point de vue curatorial, je m'intéresse à des lectures subjectives, et parfois polémiques, sur des œuvres par rapport à leur intentionnalité. La forme de l'exposition autorise des raccourcis et des rapprochements qui, théoriquement, ne sont peut-être pas recevables et certainement difficiles à justifier par l'analyse. J'entends par là que ce qui pourrait s'effondrer dans l'argumentaire d'un essai peut trouver une valeur dans le contexte d'une exposition. Car une exposition montre mais ne démontre pas : on met des choses en face les unes des autres. C'est performatif plus qu'analytique. Dans «Subréel », Jim Shaw côtoyait Tacita Dean, et ce sont des artistes que tout oppose a priori, mais qui engageaient dans ce contexte un rapport ténu sur la fascination et l'ennui.

R.c. : Que pensez-vous de la notion de vertige?

F.p.: Cela ne m'intéresse pas d'évanouir le spectateur mais cela m'intéresse qu'il y pense.

R.c. : On retrouve cette idée dans l'exposition «Doubtiful - dans les plis du réel » qui se décrit comme une expérience "génératrice de réalités qui agissent comme autant de failles susceptibles de contaminer insidieusement notre entendement. »

F.p. : Je n'ai pas vu cette exposition, j'ai participé à leur catalogue, sous la forme d'un texte intitulé « Script invisible » qui est depuis devenu le titre d'une exposition (à w139, Amsterdam, au printemps 2005) prolongeant certains questionnements de «Subréel» mais qui repose sur d'autres postulats, notamment l'envie d'adapter un roman en exposition. C'est un prétexte comme un autre pour faire exposition.

\section{R.c. : Ce prétexte ne se mue-t-il pas en thématique?}

F.p. : Aujourd'hui, il est plutôt de bon ton d'être contre les expositions thématiques mais, selon moi, il n'y a pas de mauvais prétexte à une exposition. Ce qui est certain, c'est que l'ancrage thématique ne garantit en rien la qualité d'une exposition. Pour ce projet, il s'agit donc de l'adaptation d'un roman d'Adolfo Bioy Casares, L'Invention de Morel qui apparaît de façon très ténue, métaphorique, comme un possible fil directeur, un scénario invisible.

R.c. : C'est effectivement une nouvelle qui entretient des liens très étroits avec l'art et spécifiquement le rapport à l'œuvre d'art. Ce récit peut être appréhendé comme un traité 
sur l'illusion et sur certains modes de perception que l'on développe en relation au réel. Il y a aussi cette idée d'addiction à l'être aimé et au mythe de l'immortalité. Cela n'est pas étonnant que l'on s'y intéresse aujourd'hui. Qu'avez-vous choisi d'adapter pour votre exposition?

F.p.: Je n'ai gardé de la trame romanesque que les rapports entretenus par le narrateur avec les personnages qui apparaissent dans son environnement : cette volonté qu'il a d'essayer de communiquer avec eux mais qui apparaît évidemment vouée d'emblée à l'échec pour le lecteur. Cela devient, dans l'exposition, une métaphore du rapport à l'œuvre. Cette exposition rassemble des œuvres qui ont une forme de comportement que l'on pourrait interpréter comme une volonté de communication avec le spectateur mais qui, bien sûr, fonctionnent en totale autarcie. Elles renvoient ainsi le spectateur à une solitude totale par leur présence. J'avais d'abord pensé cette exposition dans un espace ouvert sur la rue. Comme ces œuvres sont des machines, il fallait qu'elles fonctionnent 24 heures sur 24. Finalement, c'est dans un tout autre type d'espace qu'elle a eu lieu et j'ai abandonné cette dimension sans doute un peu trop explicite, même si elle était séduisante. L'Invention de Morel est pour moi une œuvre très abstraite. Le décor est utilisé de manière extrêmement conceptuelle par Bioy Casares. Il se sert du terrain archétypal de l'île déserte comme motif pour y calquer quelque chose de beaucoup plus abstrait. Par ailleurs, tous ses romans sont construits ainsi. Plan d'évasion se déroule dans un contexte tropical, dans un bagne et l'on comprend progressivement que le directeur est en train d'essayer un mode expérimental de prison qui fonctionne sur la création d'une idée complètement artificielle de liberté. Il contraint physiologiquement les prisonniers avec des codes de couleur pour leur donner l'illusion qu'ils sont dans un espace ouvert alors qu'ils sont dans un espace fermé. On a l'impression de lire la description d'un Dan Graham. 
Laurent Montaron, Somniloquie

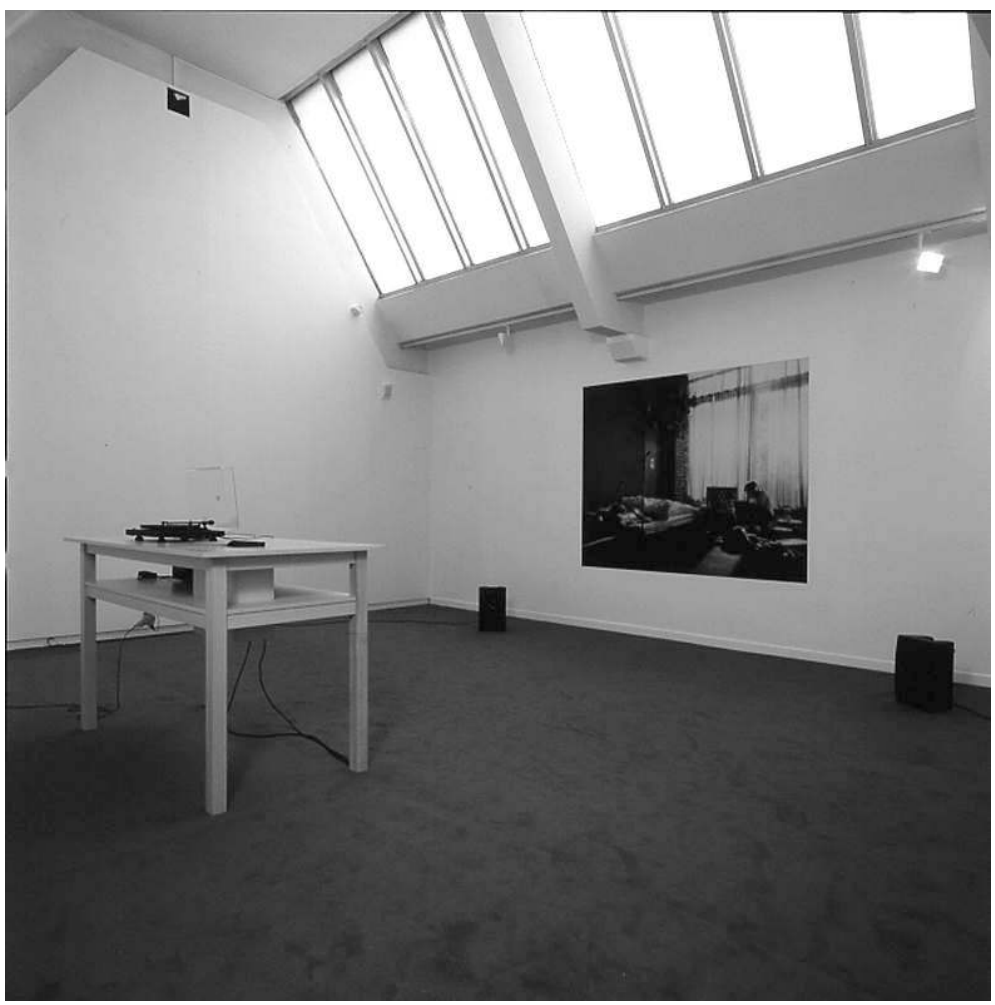

Photographie $180 \times$ 230, meuble, platine, disque vinyles, 2002 .

\section{Berdaguer-Péjus, Morphine Landscape}

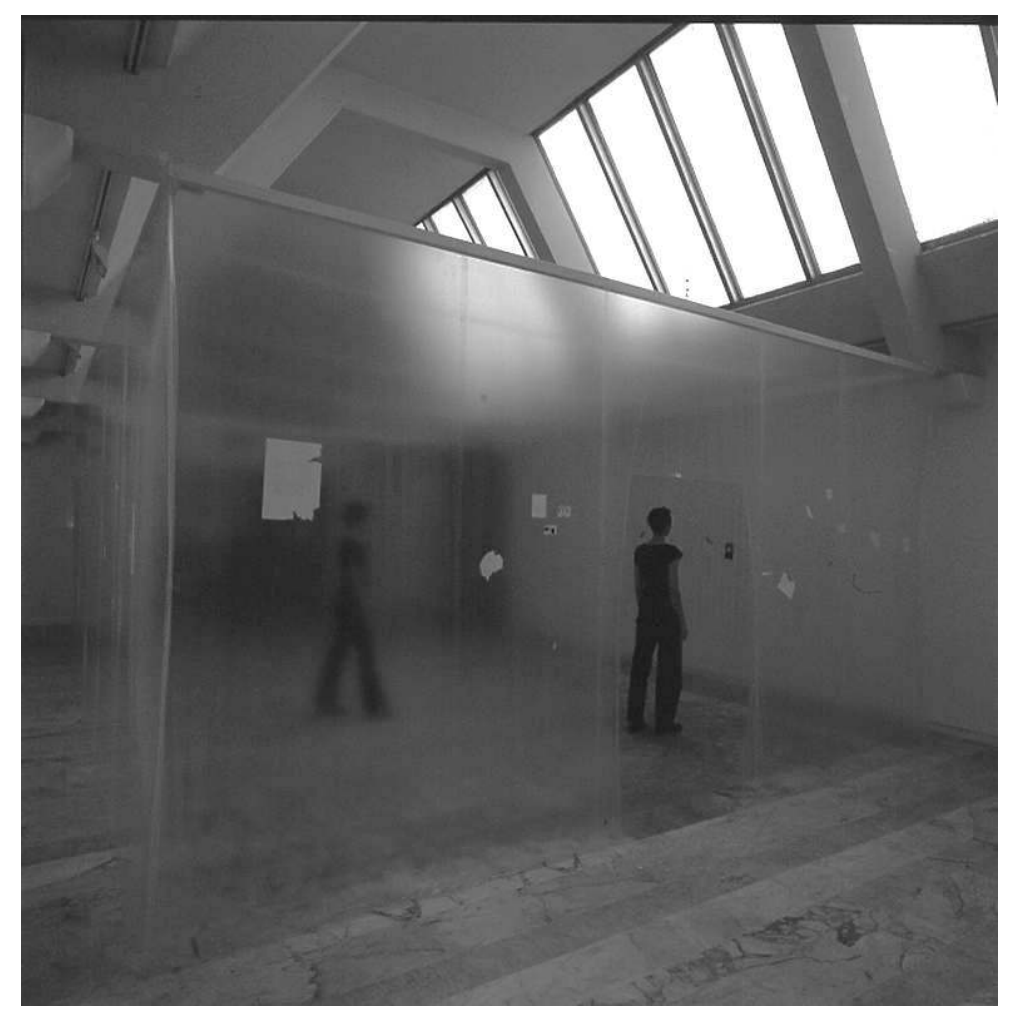

Bbois, éclairage, morphine, 2002. 


\section{RÉSUMÉS}

Depuis les années 1960, la figure du curateur est sans aucun doute au cœur des débats. À l'occasion de "Subréel », François Piron revient sur les motivations de ses choix en tant que commissaire d'exposition. On verra notamment que la posture du curateur engage le visiteur dans une lecture parfois subjective de l'art contemporain, interprétation d'autant plus palpable à l'ère du simulacre.

Since the sixties, the curator as a character is no doubt at the heart of debates. On the occasion of "Subréel", François Piron re-examines the motivations of his choices as exhibitions' curator. One can notice how the curator's attitude forces the visitor into a sometimes subjective reading of contemporary art; an all the more so tangible interpretation in an era of simulacra.

\section{AUTEURS}

\section{ROZENN CANÉVET}

Doctorante en Arts Plastiques, Sciences et Technologies des Arts à Paris 8, ater.

\section{FRANÇOIS PIRON}

Commissaire indépendant 